\title{
Pantethine and Cystamine Deplete Cystine from Cystinotic Fibroblasts via Efflux of Cysteamine-Cysteine Mixed Disulfide
}

\section{Jean DeB. Butler and Martin Zatz}

Section on Biochemical Genetics, Human Genetics Branch, National Institute of Child Health and Human Development, Unit on Biochemical Pharmacology, Laboratory of Cell Biology, National Institute of Mental Health, Bethesda, Maryland 20205

A

bstract. Children suffering from cystinosis, a genetic disease characterized by high levels of lysosomal cystine, are currently being treated with cysteamine to lower the cystine levels in their cells. In fibroblasts from these patients, cysteamine and its disulfide, cystamine, are equally effective in lowering cystine levels. We recently reported that pantethine, a dietary precursor of coenzyme A, depletes cystine from cultured cystinotic fibroblasts as effectively as cystamine. To determine the mechanism of action of pantethine, and of cystamine, we have compared the fate of $\left[{ }^{35} \mathrm{~S}\right]$ cystine-derived metabolites in the presence and absence of these agents. The results indicate that the ability of pantethine to deplete cystine resides in its being a metabolic precursor of cysteamine. Furthermore, both pantethine and cystamine act by generating the mixed disulfide of cysteamine and cysteine in the lysosomes, which is then rapidly excreted from the cells. The fall in intracellular $\left[{ }^{35} \mathrm{~S}\right]$ cystine caused by these agents was not accompanied by a comparable increase in any intracellular metabolite; rather, it could be accounted for by the appearance of mixed disulfide in the medium. There was no accumulation of mixed disulfide in the cells. Radioactivity in cytoplasmic glutathione was, however, increased by cystamine or pantethine. Thus, cysteamine (formed intracellularly in these experiments) undergoes thiol-disulfide exchange with cystine in the lysosomes, producing cysteamine-cysteine mixed disulfide and free cysteine, which enter the cytoplasm. The free cysteine is available to several pathways, including oxidation to the disulfide or the mixed disulfide, and synthesis of glutathione. The mixed disulfide is excreted from the cell, which ultimately depletes the cell of its excess cystine.

Received for publication 15 June 1983 and in revised form 2 April 1984.

The Journal of Clinical Investigation, Inc.

Volume 74, August 1984, 411-416

\section{Introduction}

Cells from patients with cystinosis contain excess (50- to 100fold) cystine, primarily in their lysosomes (1). This excess cystine is thought to be detrimental to cell function, and efforts in the clinical treatment of this genetic disease have been directed toward lowering cellular cystine content. Several aminothiols have been shown to lower intracellular cystine in cultured cells derived from patients (2). The most effective compound is cysteamine, the reduced form of cystamine, and children suffering from cystinosis are currently being treated with this compound. In fibroblasts from cystinotic patients, cystamine is as effective as cysteamine, presumably because it is reduced to cysteamine in the cytoplasm. We have recently shown that pantethine, the disulfide of pantetheine, is comparable in effectiveness to cystamine (3). Clinically, it may prove to be preferable to cysteamine.

The mechanism of action of cysteamine is thought to involve disulfide exchange in the lysosomes followed by diffusion of cysteine and cysteamine-cysteine mixed disulfide into the cytosol (2). Recent studies with isolated lysosomes support this mechanism (Gahl, W. A., F. Tietze, J. DeB. Butler, R. Steinherz, and J. D. Schulman, unpublished results). The subsequent fate of cysteine and the mixed disulfide in the cytoplasm have not been investigated. Neither cystamine nor pantethine is effective in depleting isolated lysosomes of cystine. The present experiments were designed to determine the fate of cystine in whole cells treated with cystamine and to elucidate the mechanism of action of pantethine. The results indicate that most of the cystine depleted by cystamine or pantethine leaves the cell in the form of cysteamine-cysteine mixed disulfide. Pantethine has the same mechanism of action as cysteamine; metabolism of pantethine releases cysteamine intracellularly.

\section{Methods}

Preincubation with $\left[{ }^{35} S\right]$ cystine. Skin fibroblasts were obtained from a cystinotic patient (with parental consent), and grown in tissue culture by using standard culture techniques. Cells were plated on $35-\mathrm{mm}$, 6-well plates (Costar, Data Packaging, Cambridge, MA), incubated at $37^{\circ} \mathrm{C}$ in a $5 \% \mathrm{CO}_{2}$ atmosphere; and medium (Eagle's Minimum Essential 
Medium supplemented with $10 \%$ fetal bovine serum and $2 \mathrm{mM}$ glutamine) were renewed twice weekly. At confluence, cells were washed twice with experimental medium (cystine-free Minimum Essential Medium containing $2 \mathrm{mM}$ glutamine and $1 \mathrm{mg} / \mathrm{ml}$ bovine serum albumin in place of serum). This experimental medium was used in all subsequent incubations. The cystinotic cells were loaded with $\left[{ }^{35} \mathrm{~S}\right]$ cystine (New England Nuclear, Boston, MA) by incubating them in $1.5 \mathrm{ml}$ of experimental medium that contained $\left[{ }^{35} \mathrm{~S}\right]$ cystine $(20 \mu \mathrm{Ci} / \mathrm{ml})$ for $24 \mathrm{~h}$. Extracellular radioactivity was then removed by washing the cells five times with experimental medium.

Effect of cystamine or pantethine. Plated cells containing $\left[{ }^{35} S\right]$ cystine and its metabolites were incubated for various times in $1.5 \mathrm{ml}$ of experimental medium (controls) or the same medium containing either $0.2 \mathrm{mM}$ cystamine (Sigma Chemical Co., St. Louis, MO) or $0.2 \mathrm{mM}$ pantethine (Calbiochem-Behring Corp., American Hoechst Corp., Los Angeles, CA). Addition of drug defined zero time. Three wells were used for each time point. Data are expressed per well. At each time point the distribution of nonprotein radioactivity among subcellular fractions and extracellular medium was determined. Before harvesting the cells, $1 \mathrm{ml}$ of medium was removed; protein was immediately precipitated by addition of $0.1 \mathrm{ml}$ of $30 \%$ sulfosalicylic acid; and the mixture placed on ice ( $M$ fraction). The monolayers of cells were washed three times with phosphate-buffered saline, then quickly released from the plates with $1 \mathrm{ml} 0.25 \%$ trypsin in phosphate-buffered saline, centrifuged at low speed, and washed again to remove trypsin. The cells were resuspended in $1 \mathrm{ml} 0.32 \mathrm{M}$ sucrose, disrupted by sonication for $10 \mathrm{~s}$ using a microprobe sonicator (Heat Systems-Ultrasonics, Inc., Plainview, NY), and centrifuged at $3,000 \mathrm{~g}$ for $10 \mathrm{~min}$, which gave a pellet $\left(\mathrm{P}_{1}\right.$ fraction containing debris, nuclei, and large granules) and a supernatant. This supernatant was transferred and centrifuged at $12,000 \mathrm{~g}$ for $20 \mathrm{~min}$, giving a pellet $\left(\mathrm{P}_{2}\right.$ fraction containing lysosomes and mitochondria) and a supernatant ( $S$ fraction). $P_{1}$ and $P_{2}$ pellets were resuspended by sonication in $1 \mathrm{ml}$ of water. Bovine serum albumin $(0.1 \mathrm{ml}, 1 \mathrm{mg} / \mathrm{ml})$ was added to $1 \mathrm{ml}$ of each fraction $\left(P_{1}, P_{2}\right.$, and $\left.S\right)$ as carrier, and sulfosalicylic acid $(0.1 \mathrm{ml}, 30 \%)$ was added to precipitate proteins. All procedures after trypsinization were carried out at $4^{\circ} \mathrm{C}$, without delay. Acidified samples were centrifuged $(12,000 \mathrm{~g}$ for $5 \mathrm{~min})$ to remove precipitated proteins and an aliquot of each $(200 \mu \mathrm{l})$ was counted to determine nonprotein radioactivity. Data represent total nonprotein radioactivity in fractions calculated from means of three samples.

Identification of radioactive products. On the basis of the results obtained measuring total nonprotein radioactivity, samples from two time points were chosen for identification of $\left[{ }^{35} S\right]$ cystine-derived products. Triplicate samples from each fraction (sulfosalicylic acid supernatants of $M, P_{2}$, and $S$ ) from the 20- and 40-min points in the cystamine experiment, and the 40 - and 60 -min points in the pantethine experiment, were pooled, respectively, and $20-\mu \mathrm{l}$ aliquots were spotted on $3 \mathrm{MM}$ Whatman chromatography paper (Whatman Laboratory Products, Inc., Whatman Paper Div., Clifton, NJ). $\left[{ }^{35}\right.$ S]Cystine-derived products were separated by high voltage electrophoresis in a High Voltage Electrophorator, model D, Gilson Medical Electronics (Middleton, WI) at 4 $\mathrm{kV}$ for $1 \mathrm{~h}$ in $7.4 \%$ formic acid. Standards were run on each paper and stained with ninhydrin $(0.25 \%$ in acetone) to locate the products. Standards were obtained from Sigma Chemical Co. or synthesized by the method of Eriksson and Eriksson (4). Pantetheine-cysteine mixed disulfide was synthesized by mixing equimolar quantities of pantethine and cysteine in sodium phosphate buffer $(10 \mathrm{mM}, \mathrm{pH} 7.4)$. The paper chromatograms were cut into $1-\mathrm{cm}$ strips and counted.

Hexosaminidase activity in the medium was assayed by the method of Rome and Crain (5).

\section{Results}

Under the conditions used, the cystinotic fibroblasts contained $\sim 3 \times 10^{6} \mathrm{cpm}$ of nonprotein radioactivity after $24 \mathrm{~h}$ incubation with ${ }^{35}$ S]cystine. Upon further incubation in the absence of $\left[{ }^{35}\right.$ S $]$ cystine (or cystine), control cells slowly lost radioactivity to the medium (Fig. 1, upper). The increase in extracellular
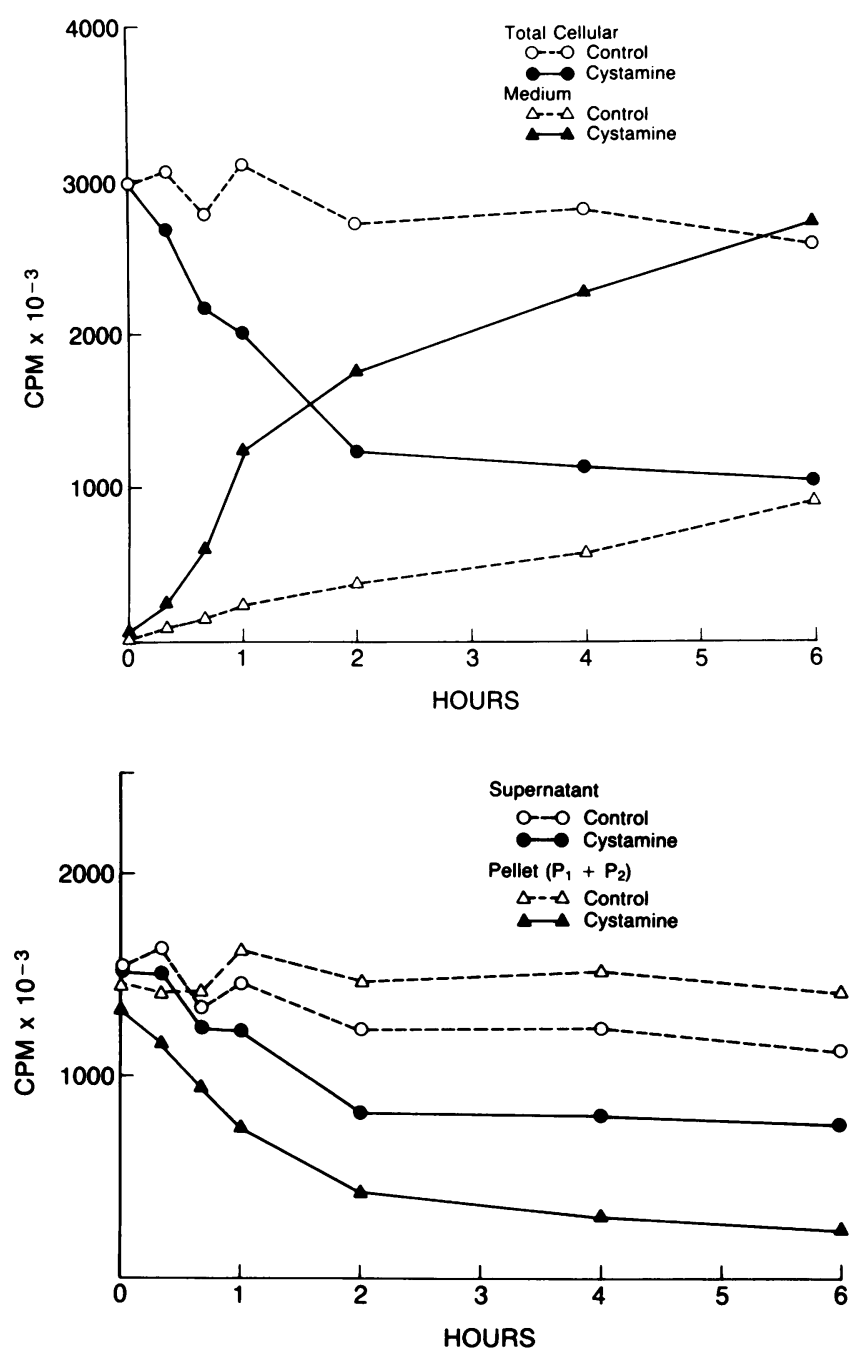

Figure 1. Effect of cystamine on $\left[{ }^{35} \mathrm{~S}\right]$ cystine-derived nonprotein radioactivity in cystinotic fibroblasts. Cells were incubated with $\left.{ }^{[35} \mathrm{S}\right]$ cystine for $24 \mathrm{~h}$ and then washed free of extracellular radioactivity. They were further incubated in the absence (control) or the presence of $0.2 \mathrm{mM}$ cystamine. At the times indicated, $\left[{ }^{35} \mathrm{~S}\right]$ cystine-derived nonprotein radioactivity was measured in the medium and subcellular fractions as described in Methods. Data have been corrected to reflect total radioactivity in the fractions. (Upper) Radioactivity in the cells $\left(P_{1}+P_{2}+S\right)$ and in the medium is plotted. (Lower) Radioactivity in the particulate $\left(P_{1}+P_{2}\right)$ fractions and in the supernatant is plotted. 
radioactivity was essentially linear with time, reaching $\sim 25 \%$ of the total present after $6 \mathrm{~h}$. In contrast, cells treated with cystamine lost radioactivity to the medium much more rapidly. More than $50 \%$ of the nonprotein radioactivity appeared in the medium within $2 \mathrm{~h}$. (Since media were collected before harvesting, neither the amounts nor the composition of $\left[{ }^{35} \mathrm{~S}\right]$ cystinederived compounds in the medium were affected by the procedures used on the cells.) It should be noted that the total nonprotein radioactivity in both control and cystamine-treated cells remained essentially unchanged, i.e., there was no substantial transfer of radioactivity to sulfosalicylic acid-precipitable material. Thus, the effect of cystamine was to markedly accelerate the efflux of $\left[{ }^{35} \mathrm{~S}\right]$ cystine-derived radioactivity out of the cells. The loss of radioactivity from the cells can be accounted for by its appearance in the medium at each time point. The rate of efflux in the presence of cystamine was nonlinear, decreasing progressively as the cells were depleted of nonprotein radioactivity.

The effect of cystamine appeared strongest on the particulate fractions. Cells treated with cystamine for $2 \mathrm{~h}$ lost 79 and $65 \%$ of their nonprotein radioactivity in $P_{1}$ and $P_{2}$, respectively, relative to controls. Results from $P_{1}$ and $P_{2}$ were similar at all time points, and have been pooled in Fig. 1 (lower). Particulate fractions in the control cells remained substantially unchanged with time. Most of the loss of nonprotein radioactivity in the control cells appeared to derive from fraction S. Cystamine increased this loss from the soluble fraction (34\% after $2 \mathrm{~h}$, relative to controls). This figure may be misleadingly low, however, since the electrophoresis data indicated a higher proportion of unaffected material (presumably peptides) in the soluble fraction than in the particulate fractions.

A second experiment using the same design was performed in order to compare the action of pantethine to that of cystamine. As illustrated in Fig. 2, pantethine's effects on nonprotein radioactivity derived from $\left[{ }^{35} \mathrm{~S}\right]$ cystine were quite similar to those of cystamine. The action of pantethine appeared to be a bit slower than that of cystamine; $50 \%$ reduction of cellular radioactivity was not achieved until $\sim 4 \mathrm{~h}$. Depletion of radioactivity from cellular compartments was accompanied by the appearance of radioactivity in the medium. After $2 \mathrm{~h}$ in the presence of pantethine, the cytoplasmic fraction (S) had lost $28 \%$ of its radioactivity, while the $P_{1}$ and $P_{2}$ fractions had lost 66 and $62 \%$, respectively, relative to controls. Again, the similarities between data from $P_{1}$ and $P_{2}$ warranted pooling for Fig. 2 (lower). Pantethine caused the loss of nonprotein radioactivity from each subcellular compartment, relative to controls, at all time points. Radioactivity lost from the cells could be accounted for by concomitant appearance of radioactivity in the medium.

Samples of each fraction (from the 20 - and $40-\mathrm{min}$ points in the cystamine experiment and from the $40-$ and $60-\mathrm{min}$ points in the pantethine experiment) were subjected to high voltage electrophoresis in order to identify the radioactive products derived from $\left[{ }^{35} \mathrm{~S}\right]$ cystine. These points were chosen to reflect the times of most rapid change. In interpreting the data,
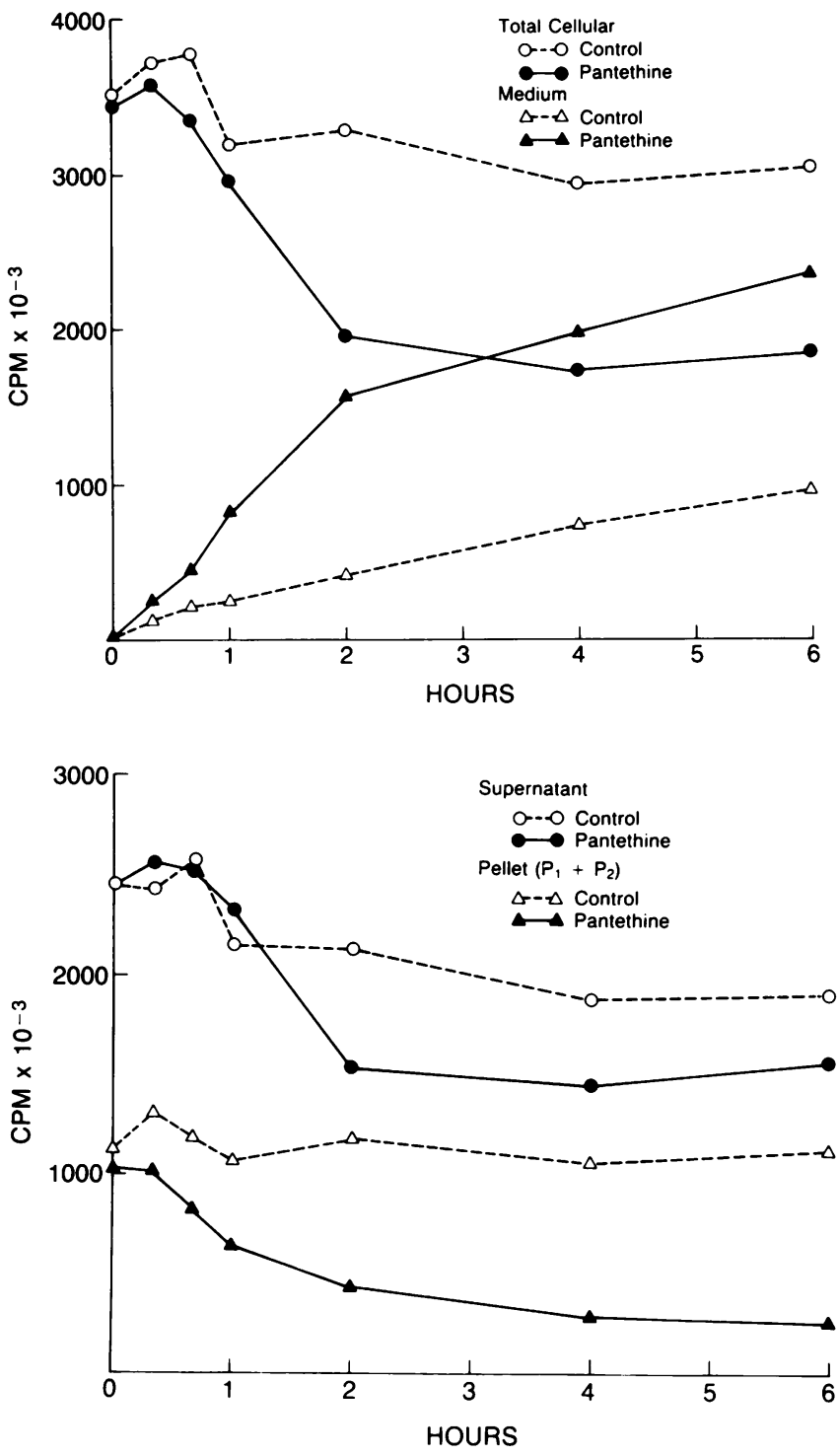

Figure 2. Effect of pantethine on $\left[{ }^{35}\right.$ S]cystine-derived nonprotein radioactivity in cystinotic fibroblasts. Experiment and figure are as in Fig. 1 except that $0.2 \mathrm{mM}$ pantethine was used in place of cystamine.

emphasis has been placed on comparisons between cystamineor pantethine-treated cells and untreated cells. The procedures used inevitably introduce some inaccuracies in the data insofar as they are expected to reflect the in vivo situation. (Since media were collected before harvesting, neither the amounts nor the composition of $\left[{ }^{35} \mathrm{~S}\right]$ cystine-derived compounds in the medium were affected by the procedures used on the cells.) Trypsinization may have caused some loss of metabolites. Lysosomal disruption upon sonication undoubtedly added radioactive cystine to the cytoplasmic (S) fraction. No attempt was made to capture free cysteine in the cytoplasm. Such systematic errors do not, how- 
ever, interfere with comparisons between groups analyzed simultaneously in the same way. Thus, these data do not accurately reflect either the levels or subcellular distribution of all $\left[{ }^{35}\right.$ S $]$ cystine-derived compounds; rather, they reveal the effects of pantethine and cystamine.

Fig. 3 shows the electrophoretic patterns obtained from samples of medium, particulate $\left(\mathrm{P}_{2}\right)$, and soluble $(\mathrm{S})$ fractions, respectively, of cystamine-treated and control cells after $40 \mathrm{~min}$. There was very little radioactivity in the medium from control
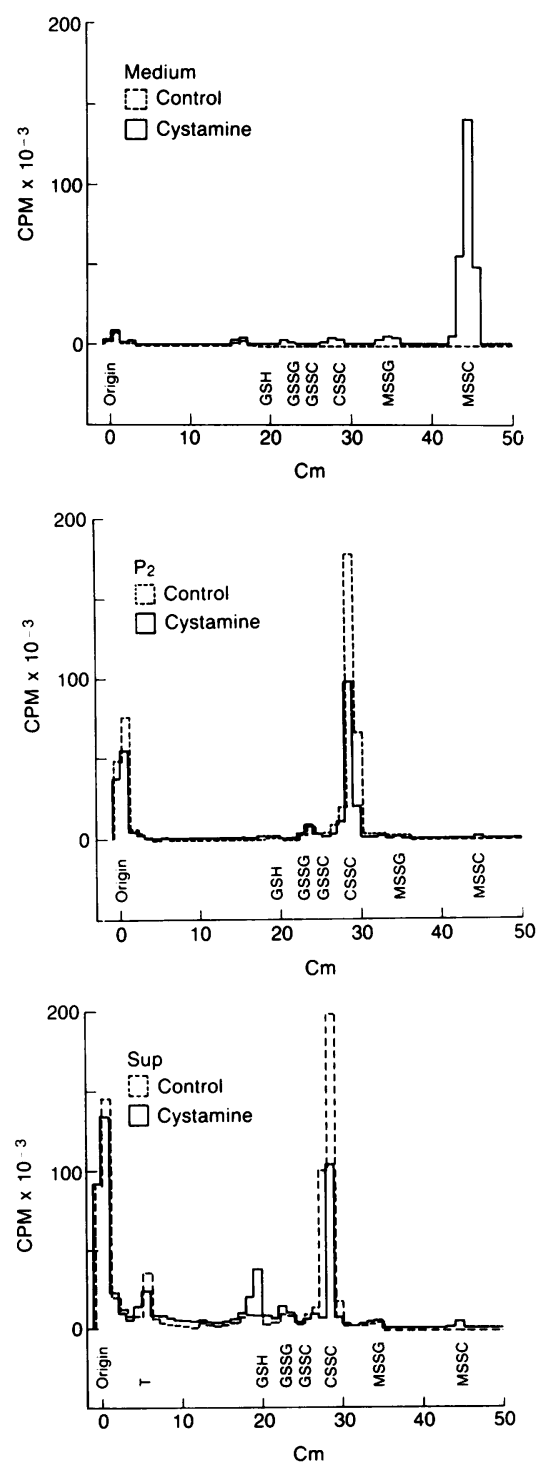

Figure 3. Effect of cystamine on $\left[{ }^{35} S\right]$ cystine-derived products in cystinotic fibroblasts. Aliquots of fractions from the 40 -min point in the experiment described in Fig. 1 were analyzed by high voltage electrophoresis. Data have been corrected to reflect total radioactivity in the fractions. (Upper) Medium. (Middle) $\mathrm{P}_{2}$. (Lower) Supernatant (Sup). Migration of standards is indicated below the figures. $\mathrm{T}$, taurine. cells, almost all of which remained at the origin. This peak was unaffected by treatment with cystamine. The striking difference between the medium from control and cystamine-treated cells was the presence of a sizeable peak corresponding to cysteaminecysteine mixed disulfide (MSSC) ${ }^{1}$ in the cystamine-treated sample. This peak accounted for $84 \%$ of the counts; smaller peaks were observed corresponding to cystine (CSSC) and cysteamineglutathione mixed disulfide (MSSG). In the $\mathrm{P}_{2}$ fraction, which should reflect lysosomes, the ${ }^{35} \mathrm{~S}$-radioactivity was predominantly (58\%) in cystine and was reduced by $48 \%$ in the cystaminetreated cells, relative to control. The peak at the origin was reduced $26 \%$ after cystamine treatment. The small peak corresponding to GSSG was unaffected. Only a trace of cysteaminecysteine mixed disulfide could be seen. In the soluble fraction (S), most of the radioactivity was in cystine (43\%), which also decreased $56 \%$ with cystamine treatment. There was a significant peak at the origin which, however, was unaffected by cystamine treatment. Material at the origin is likely to consist of peptides that were not precipitated by sulfosalicylic acid. The peak at 6 $\mathrm{cm}$, corresponding to taurine, an oxidation product of cysteine, was somewhat decreased. Slightly more cysteamine-cysteine mixed disulfide could be seen in the soluble sample from cystamine-treated cells than in the corresponding $\mathbf{P}_{2}$ fraction. There was a significant increase of radioactivity in GSH after cystamine treatment.

Electrophoretic patterns obtained from $\mathrm{M}, \mathrm{P}_{\mathbf{2}}$, and $\mathrm{S}$ fractions from the pantethine experiment were virtually indistinguishable from those obtained in the cystamine experiment. Data from the 60 -min point are illustrated in Fig. 4. The revealing feature was that pantethine caused the efflux of $\left[{ }^{35} \mathrm{~S}\right]$ cystine-derived radioactivity not as cystine or as pantetheine-cysteine mixed disulfide but as cysteamine-cysteine mixed disulfide. ${ }^{2}$ Another feature clearly observed in the pantethine experiment was a 2.5-fold increase in radioactivity in both reduced and oxidized glutathione, relative to controls, in the $\mathrm{S}$ fraction.

The possibility was considered that cystamine promotes release of disulfide directly from the lysosomes to the extracellular space by fusion with the plasma membrane. If so, such release might include lysosomal enzymes. Medium was tested for hexosaminidase (a lysosomal enzyme) using samples from the 60-min time point of the cystamine experiment. Low levels of hexosaminidase activity were detected $(12 \mathrm{nmol} / \mathrm{min}$ per 100 $\mu \mathrm{l})$, which did not differ between medium from control and cystamine-treated cells. This result fails to support, but does not rule out, secretion of mixed disulfide directly from the lysosomes to the medium.

1. Abbreviations used in this paper: CSSC, cystine; MSSC, cysteaminecysteine mixed disulfide; MSSG, cysteamine-glutathione mixed disulfide. 2. Pantetheine-cysteine mixed disulfide standard migrated somewhat less than GSH. A small amount of radioactivity was detected in the medium from pantethine treated cells at this location. However, comparable amounts were seen at the corresponding location in media from control and cystamine treated cells. It is possible, therefore, that this small peak reflects another $\left[{ }^{35} \mathrm{~S}\right]$ cystine derived product. 

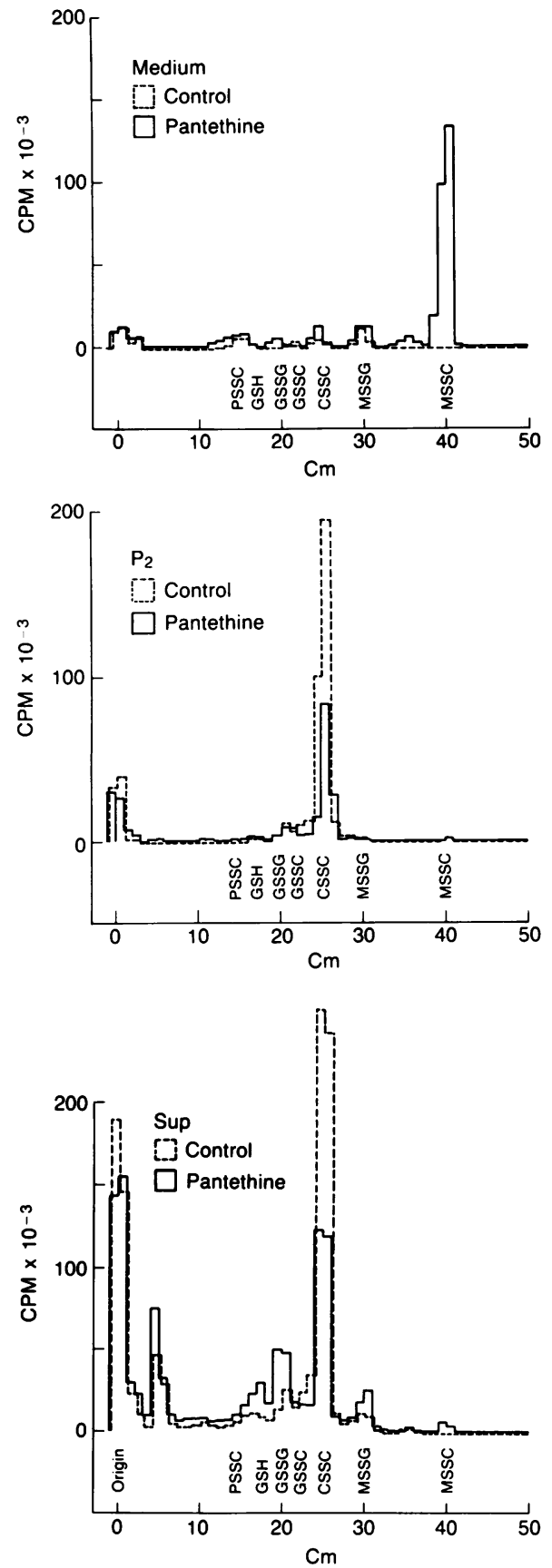

Figure 4. Effect of pantethine on $\left[{ }^{35} \mathrm{~S}\right] \mathrm{cystine-derived} \mathrm{products} \mathrm{in} \mathrm{cys-}$ tinotic fibroblasts. Aliquots of fractions from the 60 -min point in the experiment described in Fig. 2 were analyzed by high voltage electrophoresis. Data, figure, and standards are as in Fig. 3. PSSC, pantetheine-cysteine mixed disulfide.

\section{Discussion}

The data indicate that most of the cystine depleted by cystamine leaves the cell. There was no substantial increase in cystine metabolites or "disappearance" of radioactivity into sulfosalicylic acid-precipitable material. Virtually all of the reduction in intracellular radioactivity could be accounted for by the appearance of cysteamine-cysteine mixed disulfide in the medium. Since no attempt was made to capture metabolites in their reduced form (e.g., by addition of $N$-ethylmaleimide), it was possible that the cysteamine-cysteine mixed disulfide was formed after efflux of cysteine by its reaction with the cystamine in the medium. However, efflux of cysteine and thiol-disulfide exchange cannot account for the predominance of cysteamine-cysteine mixed disulfide in the medium after treatment of cells with pantethine. Taken together the experiments strongly indicate the formation of cysteamine-cysteine intracellularly followed by its efflux into the medium.

The experiments also demonstrate a convergence in the mechanisms of action of pantethine and cystamine. The reduced form of pantethine, pantetheine, is a known (indeed, the only known) physiologic precursor for cysteamine (6). Thus, the results are most readily interpreted as follows: The disulfides cystamine and pantethine enter the cell and are reduced (by glutathione) to the free thiols cysteamine and pantetheine, respectively. Pantetheine is then cleaved to pantothenic acid and cysteamine by the enzyme pantetheinase (7). Cystinotic cells have normal levels of pantetheinase (8). Cysteamine enters the lysosome and reacts with the disulfide cystine to form cysteamine-cysteine mixed disulfide and cysteine. Both of these products leave the lysosome and enter the cytoplasm. There, free cysteine can react with cytoplasmic cysteamine or cystamine to form more mixed disulfide. It can also oxidize to cystine, or enter the glutathione pool. Glutathione turnover may well be increased consequent to its use in reducing cystamine or pantethine. Entry of the released $\left[{ }^{35} S\right]$ cysteine into the glutathione pool probably accounts for the increase in radioactive glutathione observed after treatment with cystamine or pantethine.

Cysteamine-cysteine mixed disulfide appears rapidly in the medium. There was no accumulation of this material in the cells. One possibility is that the mixed disulfide is secreted directly into the medium. This seems unlikely for a number of reasons. First, extracellular hexosaminidase (a lysosomal enzyme) did not increase after cystamine treatment. Second, transfer of cysteine from the lysosomes to the cytoplasm is suggested by the increase in radioactive glutathione in the cytoplasm and the absence of radioactive cysteine or cystine in the medium. Finally, cysteamine induced efflux of free cysteine and formation of mixed disulfide have been observed using isolated lysosomes (Gahl, W. A., F. Tietze, J. DeB. Butler, R. Steinherz, and J. D. Schulman, unpublished results). Another possibility is that the mixed disulfide is rapidly transported out of the cell. Evidence for lysosomal transport of cystine by carrier-mediated (9) and energy-dependent $(10)$ processes have been presented. Whether efflux of cysteamine-cystine mixed disulfide from lysosomes and cells is an active or passive process remains to be determined.

Cysteamine is currently used to treat children with cystinosis. Its clinical effectiveness is limited by its systemic toxicity (11). 
Pantethine is absorbed intact, is relatively nontoxic systematically, and has been used clinically in patients with hyperlipidemia (12). The results described here indicate that pantethine generates intracellular cysteamine to remove excess cystine from cystinotic cells. Its relative safety and intracellular targeting may provide a preferable alternative for the treatment of cystinosis.

\section{References}

1. Schneider, J. A., and J. D. Schulman. 1982. Cystinosis. In The Metabolic Basis of Inherited Disease. J. B. Stanbury, J. B. Wyngaarden, D. S. Fredrickson, J. L. Goldstein, and M. S. Brown, editors. McGrawHill Book Co., Inc. New York. Fifth ed. 1844-1866.

2. Thoene, J. G., R. G. Oshima, J. C. Crawhill, D. L. Olsen, and J. A. Schneider. 1976. Intracellular cystine depletion by aminothiols in vitro and in vivo. J. Clin. Invest. 58:180-189.

3. Butler, J. DeB., and M. Zatz. 1983. Pantethine depletes cystinotic fibroblasts of cystine. J. Pediatr. 102:796-798.

4. Ericksson, B., and S. A. Ericksson. 1967. Synthesis and characterization of the L-cysteine-glutathione mixed disulfide. Acta Chim. Scand. 21:1304-1312.
5. Rome, L. H., and L. R. Crain. 1981. Degradation of mucopolysaccharide in intact isolated lysosomes. J. Biol. Chem. 256:10763-10768.

6. Abiko, Y. 1967. Investigations on pantothenic acid and its related compounds. J. Biochem. 61:290-299.

7. Ono, S., K. Kameda, and Y. Abiko. 1974. Metabolism of pantethine in the rat. J. Nutr. Sci. Vitaminol. 20:203-213.

8. Orloff, S., J. DeB. Butler, D. Towne, A. B. Mukherjee, and J. D. Schulman. 1981. Pantetheinase activity and cysteamine content in cystinotic and normal fibroblasts and leucocytes. Pediatr. Res. 15:10631067.

9. Gahl, W. A., N. Basham, F. Tietze, I. Bernadini, and J. D. Schulman. 1982. Cystine transport is defective in isolated leucocyte lysosomes from patients with cystinosis. Science (Wash. DC). 217:1263-1265.

10. Jonas, A. J., M. L. Smith, and J. A. Schneider. 1982. ATPdependent lysosomal cystine efflux is defective in cystinosis. J. Biol. Chem. 257:13185-13188.

11. Corden, B. J., J. D. Schulman, J. A. Schneider, and J. G. Thoene. 1981. Adverse reactions to oral cysteamine use in nephropathic cystinosis. Dev. Pharmacol. Ther. 3:25-30.

12. Avogaro, P., G. B. Bon, and M. Fusello. 1983. Effect of pantethine on lipids, lipoproteins and apolipoproteins in man. Curr. Ther. Res. 33:488-493. 